PROCEEDINGS OF THE

AMERICAN MATHEMATICAL SOCIETY

Volume 134, Number 6, Pages 1791-1802

S 0002-9939(05)08160-8

Article electronically published on October 28, 2005

\title{
ONE-DIMENSIONAL METRIC FOLIATIONS ON THE HEISENBERG GROUP
}

\author{
MARIUS MUNTEANU \\ (Communicated by Jon G. Wolfson)
}

\begin{abstract}
We prove that the one-dimensional metric foliations on the $(2 n+1)$-dimensional Heisenberg group equipped with a left invariant metric are homogeneous.
\end{abstract}

\section{INTRODUCTION}

A $k$-dimensional foliation $\mathcal{F}$ on a differentiable manifold $M$ is a partition of $M$ into $k$-dimensional (immersed) submanifolds called leaves. If $M$ is endowed with a Riemannian structure, $\mathcal{F}$ is called metric if its leaves are locally equidistant.

Let $G$ be a connected subgroup of the isometry group of $M$. The orbits of $G$ acting on $M$ are equidistant and, if they have the same dimension, one gets a homogeneous foliation. This is the primary source for metric foliations.

The natural question to be asked is whether all metric foliations are homogeneous. Even though, in this generality, the answer is negative (for examples, see [2]), it seems that a large degree of symmetry does imply homogeneity, at least for manifolds with nonnegative curvature.

Two of the most important results in this direction assert homogeneity of onedimensional metric foliations on spaces of constant nonnegative curvature ([2]) and of metric fibrations of arbitrary dimension on Euclidean spaces (4]).

Since from a Lie algebra perspective the Heisenberg group is the closest to the Euclidean space, one may ask whether the same property holds in this case. In this paper we provide a positive answer for the one-dimensional foliations. An interesting consequence is that on the three-dimensional Heisenberg group, using a result of G. Walschap on codimension one metric foliations on $H^{2 n+1}$ ([10]), one may, indeed, claim that all metric foliations are homogeneous. We also mention that the same homogeneity property is known to hold on three-dimensional nilmanifolds ([9]).

First, we review some basic facts about metric foliations and the geometry of the Heisenberg group with a left invariant metric.

\section{Generalities on metric foliations}

For a detailed treatment of metric foliations and Riemannian submersions, the reader is referred to [6], 7], and [8].

Received by the editors December 15, 2003 and, in revised form, December 31, 2004.

2000 Mathematics Subject Classification. Primary 53C12; Secondary 22E25, 57R30.

(C)2005 American Mathematical Society

Reverts to public domain 28 years from publication 
Let $M, B$ be differentiable manifolds with $\operatorname{dim}(M) \geq \operatorname{dim}(B)$ and let $\pi: M \rightarrow B$ be a submersion, i.e., $\pi$ is a surjective differentiable map of maximal rank. For any $b \in B, \pi^{-1}(b)$ is a submanifold of $M$ of dimension $\operatorname{dim}(M)-\operatorname{dim}(B)$. Consequently, for each $m \in M$ one has a decomposition of the tangent space $M_{m}$ into a vertical subspace $\mathcal{V}_{m}$ tangent to $\pi^{-1}(\pi(m))$ and a horizontal space $\mathcal{H}_{m}=\mathcal{V}_{m}^{\perp}$.

A differentiable map $\pi: M \rightarrow B$ is called a Riemannian submersion if $\pi$ is a submersion and $\pi_{*}$ preserves the length of horizontal vectors, that is, $\left|\pi_{*} x\right|=|x|$ for all $m \in M, x \in \mathcal{H}_{m}$.

One can easily check that every Riemannian submersion $\pi: M \rightarrow B$ determines a metric foliation whose leaves are given by the preimages of points in $B$. The converse is also true locally. Thus, the following definitions and remarks, formulated in the language of Riemannian submersions, can be extended for metric foliations.

As noted in [6], the crucial role in the understanding of a Riemannian submersion is played by the integrability tensor $A$ and the second fundamental form $S$ given by

$$
\begin{aligned}
& A: \mathcal{H} \times \mathcal{H} \rightarrow \mathcal{V}, \quad A_{X} Y=\left(\nabla_{X} Y\right)^{v}, \\
& S: \mathcal{H} \times \mathcal{V} \rightarrow \mathcal{V}, \quad S_{X} V=-\left(\nabla_{V} X\right)^{v} .
\end{aligned}
$$

The mean curvature form $\kappa$ is the horizontal 1-form on $M$ defined by $\kappa(E)=$ $\operatorname{tr} S_{E^{h}}$. If the leaves are one dimensional, $\kappa$ becomes $\kappa(X)=\left\langle S_{X} V, V\right\rangle$, where $X \in \mathcal{H}$ and $V \in \mathcal{V}$ with $|V|=1$.

A horizontal vector field $X$ on $M$ is called basic if $\pi_{*} X=\tilde{X} \circ \pi$, where $\tilde{X}$ is a vector field on $B$. In the special case that $X$ is a vector field along $\pi^{-1}(b), b \in B$, $X$ will still be called basic if $\pi_{*} X_{m}=\tilde{X}_{b}$, for all $m \in \pi^{-1}(b)$. Finally, a horizontal 1 -form on $M$ is called basic if the dual vector field is basic.

Let $\tilde{\gamma}$ be a geodesic in $B$ with $\dot{\tilde{\gamma}}(0)=\tilde{x}$ and let $X$ be the unique basic vector field along $\pi^{-1}(b)$ with $\pi_{*} X=\tilde{x}$. For each $m \in \pi^{-1}(b)$ consider the geodesic $\gamma_{m}$ in $M$ starting at $m$ in direction $X_{m}$. This way we can define a diffeomorphism $h_{\tilde{\gamma}}^{t}: \pi^{-1}(\tilde{\gamma}(0)) \rightarrow \pi^{-1}(\tilde{\gamma}(t))$, called the holonomy displacement. Note that every curve $c$ in $\pi^{-1}(\tilde{\gamma}(0))$ gives rise to a geodesic variation $H_{\tilde{\gamma}}$ of $\gamma:=\gamma_{c(0)}$ given by $H_{\tilde{\gamma}}(t, s):=h_{\tilde{\gamma}}^{t}(c(s))$. The vertical vector field $J(t)=\left.\left(H_{\tilde{\gamma}}\right)_{*}\left(\frac{\partial}{\partial s}\right)\right|_{(t, 0)}$ is called a holonomy Jacobi field and

$$
J^{\prime}=J^{\prime v}+J^{\prime h}=-S_{\dot{\gamma}} J-A_{\dot{\gamma}}^{*} J,
$$

where $A_{\dot{\gamma}}^{*}$ is the adjoint of $A_{\dot{\gamma}}$. Note that if the leaves have dimension one and if $|J(0)|=|\dot{c}(0)|=1$, then

$$
\left\langle J(0), J^{\prime}(0)\right\rangle=-\left\langle J(0), S_{\dot{\gamma}(0)} J(0)\right\rangle=-\kappa(\dot{\gamma}(0)) .
$$

The following theorems are of key importance in the understanding of onedimensional metric foliations in their relationship to homogeneous metric foliations.

Theorem (2]). A one-dimensional metric foliation $\mathcal{F}$ on a manifold with constant nonnegative sectional curvature is homogeneous iff $\kappa$ is basic and closed.

Theorem ([1]). Let $\mathcal{F}$ be a one-dimensional metric foliation on a manifold with bounded sectional curvature. If $\kappa$ is basic, then it is also closed.

Thus, in order to show that a metric foliation on a space with bounded sectional curvature is homogeneous, it is enough to show that the mean curvature form $\kappa$ is basic. 


\section{Geometry of the Heisenberg group WITH A LEFT INVARIANT METRIC}

Let $\mathcal{V}$ be a $2 n$-dimensional vector space and let $\left\{X_{1}, \ldots, X_{n}, Y_{1}, \ldots, Y_{n}\right\}$ be any basis of $\mathcal{V}$. Let $\mathcal{Z}$ be a one-dimensional vector space spanned by some element $Z$. The bracket relations

$$
\left[X_{i}, Y_{i}\right]=-\left[Y_{i}, X_{i}\right]=Z, 1 \leq i \leq n,
$$

and all other brackets zero define a Lie algebra structure on $\mathfrak{h}=\mathcal{V} \oplus \mathcal{Z}$. With this structure, $\mathfrak{h}$ is called the $(2 n+1)$-dimensional Heisenberg algebra, and the corresponding simply connected Lie group $H^{2 n+1}$ is called the $(2 n+1)$-dimensional Heisenberg group.

It is well known that the Lie group exponential map exp $: \mathfrak{h} \rightarrow H$ is a diffeomorphism. Moreover, the Lie group multiplication is given by

$$
\exp (X) \cdot \exp (Y)=\exp \left(X+Y+\frac{1}{2}[X, Y]\right)
$$

Consider a positive definite inner product $\langle$,$\rangle on \mathfrak{h}$ and define the skew-symmetric transformation

$$
\begin{aligned}
j=j(Z): \mathcal{Z}^{\perp} & \rightarrow \mathcal{Z}^{\perp}, \\
X & \rightarrow(\operatorname{ad} X)^{*} Z,
\end{aligned}
$$

where $\operatorname{ad} X: \mathfrak{h} \rightarrow \mathfrak{h}$ is given by ad $X(Y)=[X, Y]$ and $(\operatorname{ad} X)^{*}$ is the adjoint of ad $X$. Note that $\langle j X, Y\rangle=\langle[X, Y], Z\rangle$. Also, if $\left|X_{i}\right|=\left|Y_{i}\right|=|Z|=1,1 \leq i \leq n$, then $j^{2}=-I d$.

Now extend $\langle$,$\rangle to a left invariant metric on H$; this way any left translation $L_{a}\left(L_{a}(b):=a \cdot b\right)$ becomes an isometry. It is well known that, with respect to such metrics, $H^{2 n+1}$ has bounded sectional curvature. From now on, we will identify as needed a left invariant vector field with the corresponding vector in the tangent space to $H$ at $e$.

3.1. The geodesics. The geodesic equations are obtained in [1] in the more general context of two-step nilpotent Lie groups with a left invariant metric. Here we will only summarize some important properties for the geodesics of the Heisenberg group. The following proposition gives the equation of a geodesic starting at $e$. Since the isometry group $\operatorname{Iso}\left(H^{2 n+1}\right)$ is transitive, any other geodesic can be obtained by left translating the geodesic above.

Proposition. Let $\gamma$ be a unit speed geodesic with $\gamma(0)=e$ and $\dot{\gamma}(0)=\cos \theta Z+$ $\sin \theta X$, where $X \perp \mathcal{Z}, Z \in \mathcal{Z}$, and $|X|=|Z|=1$.

(i) If $\theta=\pi / 2$, then $\gamma(t)=\exp (t X)$.

(ii) If $\theta \in(0, \pi / 2)$, then $\gamma(t)=\exp (X(t)+Z(t))$, where

$$
\begin{gathered}
X(t)=[\cos (t \cos \theta)-1] \tan \theta j^{-1} X+\sin (t \cos \theta) \tan \theta X, \\
Z(t)=\left[t\left(1+\frac{1}{2} \tan ^{2} \theta\right) \cos \theta-\frac{1}{2} \sin (t \cos \theta) \tan ^{2} \theta\right] Z .
\end{gathered}
$$

(iii) If $\theta=0$, then $\gamma(t)=\exp (t Z)$.

Observations. 1 . If $\dot{\gamma}(0)$ is orthogonal to the central direction, then $\gamma$ minimizes the distance between any two of its points, i.e., $\gamma$ is a line. Actually, these are the only lines through $e$. 
2. In the second case above, $\gamma$ minimizes up to the first conjugate point which occurs at $t=2 \pi / \cos \theta$. Moreover,

$$
\dot{\gamma}(t)=\left(L_{\gamma(t)}\right)_{*}\left[-\sin (t \cos \theta) \sin \theta j^{-1} X_{i}+\cos (t \cos \theta) \sin \theta X_{i}+Z \cos \theta\right] .
$$

As a consequence, for any $N \in \mathbb{Z}$,

$$
\dot{\gamma}(2 \pi N / \cos \theta)=\left(L_{\gamma(2 \pi N / \cos \theta)}\right)_{*}(\dot{\gamma}(0)) .
$$

This property plays a significant role in determining the Riemannian foliations on $H^{2 n+1}$. Another interesting property is that any two unit speed geodesics $\gamma_{1}$ and $\gamma_{2}$ with $\gamma_{1}(0)=\gamma_{2}(0)=e$ and making the same angle $\theta$ with $\mathcal{Z}^{\perp}$ will intersect at $\gamma_{1}(2 \pi N / \cos \theta)=\gamma_{2}(2 \pi N / \cos \theta)=\exp \left(2 \pi N\left(1+\frac{1}{2} \tan ^{2} \theta\right)\right) Z$, for any $N \in \mathbb{Z}$.

3. If $\dot{\gamma}(0)=Z$, then $\gamma$ minimizes up to the first conjugate point which occurs at $2 \pi$. From now on, every geodesic tangent to the central direction will be called central.

4. Every geodesic makes a constant angle with the central direction.

3.2. The Jacobi vector fields. In this section we describe the Jacobi vector fields along geodesics $\gamma$ in $H^{2 n+1}$. We use slightly more general versions of the formulas in 5 .

Let $\gamma$ be a geodesic in $H^{2 n+1}$ with $\gamma(0)=e$ and let $J$ be a Jacobi vector field along $\gamma$. Depending on the angle made by $\gamma$ with the central direction we have the following possibilities:

(i) If $\dot{\gamma}(0)=X,|X|=1, X \in \mathcal{Z}^{\perp}$, then any Jacobi field along $\gamma$ has the form

$$
J(t)=f(t) Z \circ \gamma(t)+g(t) Y \circ \gamma(t)+\sum_{i=2}^{n}\left(f_{i}(t) X_{i} \circ \gamma(t)+g_{i}(t) Y_{i} \circ \gamma(t)\right),
$$

where $X, Y, X_{i}$ and $Y_{i}$ are left invariant vector fields whose values at $e$ (also denoted by $X, Y, X_{i}$, and $Y_{i}$, respectively) are defined such that $Y=$ $j(X), Y_{i}=j\left(X_{i}\right), i=2, \ldots, n$, and $\left\{X, Y, X_{2}, \ldots, X_{n}, Y_{2}, \ldots, Y_{n}, Z\right\}$ is an orthonormal basis of $\mathfrak{h}_{2 n+1}$.

The coefficients $f, g, f_{i}$, and $g_{i}$ are given by the following formulas:

$$
\begin{gathered}
f(t)=f(0)+t f^{\prime}(0)+\frac{1}{2} g^{\prime}(0) t^{2}+\frac{1}{6}\left(g(0)-f^{\prime}(0)\right) t^{3}, \\
g(t)=g(0)+t g^{\prime}(0)+\frac{1}{2}\left(g(0)-f^{\prime}(0)\right) t^{2}, \\
f_{i}(t)=f_{i}(0)+t f_{i}^{\prime}(0), \quad g_{i}(t)=g_{i}(0)+t g_{i}^{\prime}(0), \quad i=2, \ldots, n .
\end{gathered}
$$

(ii) If $\dot{\gamma}(0)=\cos \theta Z+\sin \theta X_{1} \in \mathcal{Z} \oplus \mathcal{Z}^{\perp},\left|X_{1}\right|=|Z|=1, \theta \in(0, \pi / 2)$, construct an orthonormal basis $\left\{X_{1}, \ldots, X_{n}, Y_{1}=j X_{1}, \ldots, Y_{n}=j X_{n}\right\}$ of $\mathcal{Z}^{\perp}$. Any Jacobi field along $\gamma$ has the form

$$
J=\sum_{k=1}^{n}\left(a_{k} E_{k}+\bar{a}_{k} \bar{E}_{k}\right)
$$

where

$$
\begin{aligned}
& E_{1}(t)=\cos \theta e^{t j(Z \cos \theta)} X_{1} \circ \gamma(t)-\sin \theta Z \circ \gamma(t), \\
& \bar{E}_{1}(t)=e^{t j(Z \cos \theta)} Y_{1} \circ \gamma(t)
\end{aligned}
$$


while for $k \geq 2$,

$$
\begin{aligned}
E_{k}(t) & =e^{t j(Z \cos \theta)} X_{k} \circ \gamma(t), \\
\bar{E}_{k}(t) & =e^{t j(Z \cos \theta)} Y_{k} \circ \gamma(t) .
\end{aligned}
$$

Notation:

$$
e^{t j(Z \cos \theta)}:=\sum_{i=0}^{\infty} \frac{t^{i}}{i !} j^{i}(Z \cos \theta)=\cos (t \cos \theta) I+\sin (t \cos \theta) j .
$$

For $k=1$ the coefficients are given by

$$
\begin{aligned}
& a_{1}(t)=a_{1}(0)+t\left(a_{1}^{\prime}(0)+c\right)+\bar{a}_{1}^{\prime}(0) \frac{1-\cos (\cos \theta t)}{\cos ^{2} \theta}-c \frac{\sin (\cos \theta t)}{\cos \theta}, \\
& \bar{a}_{1}(t)=\bar{a}_{1}(0)+\bar{a}_{1}^{\prime}(0) \frac{\sin (\cos \theta t)}{\cos \theta}+c \frac{1-\cos (\cos \theta t)}{\cos \theta},
\end{aligned}
$$

where $c=\bar{a}_{1}(0) \tan ^{2} \theta-a_{1}^{\prime}(0)\left(1+\tan ^{2} \theta\right)$. For $k \geq 2$,

$$
\begin{aligned}
& a_{k}(t)=a_{k}(0)+\bar{a}_{k}^{\prime}(0) \frac{1-\cos (\cos \theta t)}{\cos \theta}+a_{k}^{\prime}(0) \frac{\sin (\cos \theta t)}{\cos \theta}, \\
& \bar{a}_{k}(t)=\bar{a}_{k}(0)+\bar{a}_{k}^{\prime}(0) \frac{\sin (\cos \theta t)}{\cos \theta}-a_{k}^{\prime}(0) \frac{1-\cos (\cos \theta t)}{\cos \theta} .
\end{aligned}
$$

Note that

$$
\begin{aligned}
& E_{k}(2 \pi / \cos \theta)=L_{\gamma(2 \pi / \cos \theta)_{*}} E_{k}(0), \\
& \bar{E}_{k}(2 \pi / \cos \theta)=L_{\gamma(2 \pi / \cos \theta)_{*}} \bar{E}_{k}(0),
\end{aligned}
$$

for $1 \leq k \leq n$.

(iii) If $\dot{\gamma}(0)=Z$, then

$$
J(t)=h(t) Z \circ \gamma(t)+\sum_{i=1}^{n}\left(f_{i}(t) X_{i} \circ \gamma(t)+g_{i}(t) Y_{i} \circ \gamma(t)\right)
$$

where $X_{i}, Y_{i}, i=1, \ldots, n$, are constructed as in (i) and

$h(t)=a t+b, f_{i}(t)=a_{i} \cos t+b_{i} \sin t+c_{i}, g_{i}(t)=a_{i} \sin t-b_{i} \cos t+d_{i}, i=1, \ldots, n$.

\section{The MAIN RESULTS}

In preparation for our first proposition we first prove the following:

Lemma 1. Let $\mathcal{W}$ be an $m$-dimensional inner product space, let $\mathcal{V}$ be a $k$-dimensional subspace of $\mathcal{W}, 1 \leq k<m-1$, and let $Z \in \mathcal{W}$ such that $Z$ is neither orthogonal to $\mathcal{V}$ nor contained in $\mathcal{V}$. Let $\theta_{0}$ be the angle made by $Z$ with $\mathcal{V}^{\perp}$ and let $0<\theta_{0}<$ $\theta<\pi / 2$. There exists a basis $\left\{h_{1}, h_{2}, \ldots, h_{m-k}\right\}$ of $\mathcal{V}^{\perp}$ such that $h_{i}$ makes an angle $\theta$ with $Z$, for any $1 \leq i \leq m-k$.

Proof. Let $Z^{h}$ be the orthogonal projection of $Z$ onto $\mathcal{V}^{\perp}$ and note that the conclusion of the lemma is equivalent to the existence of a basis as above for which each element of the basis makes an angle $\alpha$ with $Z^{h}$, where $\alpha=\cos ^{-1}\left(\cos \theta / \cos \theta_{0}\right)$. In order to achieve this consider a basis $\left\{\bar{h}_{1}, \bar{h}_{2}, \ldots, \bar{h}_{m-k-1}\right\}$ of $\left(\mathcal{V} \oplus \operatorname{span}\left(Z^{h}\right)\right)^{\perp}$ and let $\bar{h}_{m-k}=-\bar{h}_{1}$. It is easy to check that the set $\left\{h_{1}, h_{2}, \ldots, h_{m-k}\right\}$ with $h_{i}=\cos \alpha Z^{h}+\sin \alpha \bar{h}_{i}, 1 \leq i \leq m-k$, satisfies the requirements above. 
Proposition 1. Let $\mathcal{F}$ be a $k$-dimensional metric foliation on $H^{2 n+1}$ with vertical bundle $\mathcal{V}$. If $\gamma$ is a horizontal geodesic making an angle $\theta \in\left(0, \frac{\pi}{2}\right)$ with $\mathcal{Z}$ and if $\gamma(0)=p$, then

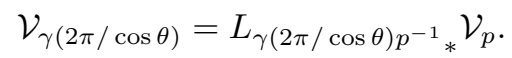

Proof. Since left translations are isometries, we may assume without loss of generality that $p=e$. Let $\theta_{0}$ be the angle made by $\mathcal{Z}$ with the horizontal space at $e$ and assume that $1 \leq k \leq 2 n-1$. If $\theta>\theta_{0}$, by the lemma above one can find a set of $2 n+1-k$ linearly independent horizontal vectors $\left\{h_{1}, h_{2}, \ldots, h_{2 n+1-k}\right\}$ at $e$ making the same angle $\theta$ with $\mathcal{Z}$. Consider the geodesics $\gamma_{i}$ starting at $e$ in direction $h_{i}$ and observe that $\gamma_{i}(2 \pi / \cos \theta)=\gamma_{j}(2 \pi / \cos \theta)$, for any $i, j=1,2, \ldots, 2 n+1-k$. Moreover, $\dot{\gamma}_{i}(2 \pi / \cos \theta)=L_{\gamma_{i}(2 \pi / \cos \theta)_{*}}\left(h_{i}\right)$ for any $1 \leq i \leq 2 n+1-k$. This implies that the set $\left\{\dot{\gamma}_{i}(2 \pi / \cos \theta), i=1,2, \ldots, 2 n+1-k\right\}$ consists of $2 n+1-k$ linearly independent vectors. Also note that since geodesics which are horizontal at one point stay horizontal for all time, the set above is actually a basis for the horizontal space at $\gamma_{i}(2 \pi / \cos \theta)$. Thus, the horizontal space at $\gamma_{i}(2 \pi / \cos \theta)$ is the left translation of the horizontal space at $e$. Consequently, the vertical spaces are in the same relation.

If $\theta=\theta_{0}$, consider the sequence $\theta_{m} \rightarrow \theta_{0}, m \geq 1$ with $\theta_{m}>\theta_{0}$ and the geodesics $\gamma_{m}$ with $\gamma_{m}(0)=e$ and making angles $\theta_{m}$ with $\mathcal{Z}$, respectively. Since $\mathcal{V}_{\gamma_{m}\left(2 \pi / \cos \theta_{m}\right)} \rightarrow \mathcal{V}_{\gamma\left(2 \pi / \cos \theta_{0}\right)}$ and $\mathcal{V}_{\gamma_{m}\left(2 \pi / \cos \theta_{m}\right)}=L_{\gamma_{m}\left(2 \pi / \cos \theta_{m}\right)_{*}} \mathcal{V}_{e}$, using a limit type of argument we may conclude that $\mathcal{V}_{\gamma\left(2 \pi / \cos \theta_{0}\right)}=L_{\gamma\left(2 \pi / \cos \theta_{0}\right)_{*}} \mathcal{V}_{e}$.

If $k=2 n$, then $\dot{\gamma}(0)$ and $\dot{\gamma}(2 \pi / \cos \theta)$ generate the horizontal spaces at $e$ and $\gamma(2 \pi / \cos \theta)$. The conclusion of the theorem holds based on the left invariance property mentioned above.

Using Proposition 1 we show that if $1 \leq k \leq 2 n-1$, then the vertical bundle of a $k$-dimensional metric foliation is left invariant along geodesics in the central direction.

Proposition 2. If $\mathcal{V}$ is the vertical bundle of a $k$-dimensional metric foliation on $H^{2 n+1}, 1 \leq k \leq 2 n-1$, then

$$
\mathcal{V}_{p \cdot \exp t Z}=L_{\exp t Z_{*}} \mathcal{V}_{p}
$$

for any $p \in H^{2 n+1}$ and $t \in \mathbb{R}$.

Proof. As before, we may assume that $p=e$. If $\mathcal{Z}$ is neither horizontal nor vertical at $e$, let $\theta_{0}$ be the angle made by $\mathcal{Z}$ with $\mathcal{H}_{e}$ and consider the following cases:

a) If $|t|>2 \pi\left(1+\frac{1}{2} \tan ^{2} \theta_{0}\right)$, there exists $\theta>\theta_{0}$ such that $\left.|t|=2 \pi\left(1+\frac{1}{2} \tan ^{2} \theta\right) Z\right)$. By Observation 2 in 3.1, there exists a horizontal geodesic $\gamma$ starting at $e$ and making an angle $\theta$ with $\mathcal{Z}$ such that $\gamma(2 \pi / \cos \theta)=\exp (t Z)$, if $t>0$, and $\gamma(-2 \pi / \cos \theta)=$ $\exp (t Z)$, if $t<0$. The result now follows from Proposition 1 .

b) If $|t| \leq 2 \pi\left(1+\frac{1}{2} \tan ^{2} \theta_{0}\right)$, consider $t_{0}<0$ with $\left|t_{0}-t\right|>4 \pi\left(1+\frac{1}{2} \tan ^{2} \theta_{0}\right)$ and note that under these conditions $t_{0}<-2 \pi\left(1+\frac{1}{2} \tan ^{2} \theta_{0}\right)$. Because of the restriction on $\left|t-t_{0}\right|$, we can find a horizontal geodesic $\gamma$ with $\gamma(0)=\exp \left(t_{0} Z\right)$ and making an angle $\theta$ with $\mathcal{Z}$ such that $\gamma(2 \pi / \cos \theta)=\exp (t Z)$. But then, by applying Proposition 1 with $p=\exp \left(t_{0} Z\right)$, we get $\mathcal{V}_{\exp (t Z)}=L_{\exp \left(t-t_{0}\right) Z_{*}} \mathcal{V}_{\exp \left(t_{0} Z\right)}$. Also, since $\left|t_{0}\right|>2 \pi\left(1+\frac{1}{2} \tan ^{2} \theta_{0}\right)$, using a) we obtain $\mathcal{V}_{\exp \left(t_{0} Z\right)}=L_{\exp \left(t_{0} Z\right)_{*}} \mathcal{V}_{e}$. Hence, $\mathcal{V}_{\exp (t Z)}=L_{\exp \left(t-t_{0}\right) Z_{*}} \mathcal{V}_{\exp \left(t_{0} Z\right)}=L_{\exp \left(t-t_{0}\right) Z_{*}} L_{\exp \left(t_{0} Z\right)_{*}} \mathcal{V}_{e}=L_{\exp (t) Z_{*}} \mathcal{V}_{e}$

If $\mathcal{Z}$ is either horizontal or vertical at $e$, then the proposition follows as a consequence of the previous case. 
It is interesting to remark that in the case of a one-dimensional foliation, Proposition 2 implies that if $\mathcal{Z}$ is vertical at $p$, then the leaf through $p$ is the geodesic through $p$ in the central direction. We will use this observation in the proof of Theorem 1.

Proposition 3. Let $\mathcal{F}$ be a one-dimensional metric foliation on $H^{2 n+1}$. If $J$ is a holonomy Jacobi vector field along a horizontal geodesic $\gamma$ making an angle $\theta \in$ $(0, \pi / 2)$ with $\mathcal{Z}$, then

$$
J(2 \pi / \cos \theta)=L_{\gamma(2 \pi / \cos \theta)_{*}} J(0) .
$$

Proof. Let $\dot{\gamma}(0)=\cos \theta Z+\sin \theta X_{1}$, where $\left|X_{1}\right|=1, X_{1} \perp \mathcal{Z}$, and let $\theta_{0}$ be the angle made by the horizontal space at $\gamma(0)$ with $\mathcal{Z}$.

If $\theta \neq \theta_{0}$, observe that, by Proposition 1, $J(0)-L_{\gamma(-2 \pi / \cos \theta)_{*}} J(2 \pi / \cos \theta)$ is vertical at $\gamma(0)=e$. By (ii) from 3.2, the holonomy Jacobi vector field $J$ along $\gamma$ can be written as

$$
J=\sum_{k=1}^{n}\left(a_{k} E_{k}+\bar{a}_{k} \bar{E}_{k}\right),
$$

with $E_{k}, \bar{E}_{k}, a_{k}$, and $\bar{a}_{k}$ given by (3)-(6). Also, by (5) and (6), $\bar{a}_{1}$ and, for $k \geq 2$, $a_{k}$ and $\bar{a}_{k}$ are periodic with period $2 \pi / \cos \theta$. Using (7), the observation above translates to $\left(a_{1}(2 \pi / \cos \theta)-a_{1}(0)\right) E_{1}(0)$ being vertical at $e$. But $E_{1}(0)$ is not vertical at $e$, since this would imply $\theta=\theta_{0}$. Consequently, $a_{1}(0)=a_{1}(2 \pi / \cos \theta)$ and $a_{1}$ is $2 \pi / \cos \theta$-periodic, thus proving the proposition in this case. Note that we also obtain that $|J(t)|$ is periodic with the same period. Moreover, $\kappa(\dot{\gamma}(t))=$ $-1 / 2\left(|J(t)|^{2}\right)^{\prime}$ is periodic as well, and $\kappa(\dot{\gamma}(0))=\kappa(\dot{\gamma}(2 \pi / \cos \theta))$.

If $\theta=\theta_{0}$, consider a sequence $\left\{h_{n}\right\}_{n \geq 1}$ of unit horizontal vectors with $h_{n} \rightarrow \dot{\gamma}(0)$. We may also assume that $\theta_{n}>\theta_{0}$, where $\theta_{n}$ is the angle made by $h_{n}$ with $\mathcal{Z}$. By a limit argument similar to the one used in Proposition 1 we obtain

$$
\kappa(\dot{\gamma}(0))=\lim _{n \rightarrow \infty} \kappa\left(\dot{\gamma}_{n}(0)\right)=\lim _{n \rightarrow \infty} \kappa\left(\dot{\gamma}_{n}\left(2 \pi / \cos \theta_{n}\right)\right)=\kappa(\dot{\gamma}(2 \pi / \cos \theta))
$$

where $\gamma_{n}$ are geodesics with $\gamma_{n}(0)=e$ and $\dot{\gamma}_{n}(0)=h_{n}$.

Now consider the holonomy Jacobi vector field $J$ along $\gamma$ with $|J(0)|=1$. Since $\theta=\theta_{0}$, we have $\dot{\gamma}(0)=\frac{1}{\left|Z^{h}\right|} Z^{h}$ and, following the notation from $3.2, J(0)=E_{1}(0)$. Consequently, $a_{1}(0)=1$, while the rest of the coefficients appearing in the formula for $J$ cancel at $t=0$ (and, by periodicity, at any integer multiple of $2 \pi / \cos \theta$ ).

By (1) and the observations above we obtain

$$
\left\langle J(0), J^{\prime}(0)\right\rangle=-\kappa(\dot{\gamma}(0))|J(0)|^{2}=a_{1}^{\prime}(0)
$$

and

$$
\begin{aligned}
\left\langle J(2 \pi / \cos \theta), J^{\prime}(2 \pi / \cos \theta)\right\rangle & =-\kappa(\dot{\gamma}(2 \pi / \cos \theta))|J(2 \pi / \cos \theta)|^{2} \\
& =a_{1}(2 \pi / \cos \theta) a_{1}^{\prime}(2 \pi / \cos \theta) .
\end{aligned}
$$

As noted above, $\kappa(\dot{\gamma}(0))=\kappa(\dot{\gamma}(2 \pi / \cos \theta))$ and the previous relations imply that $a_{1}^{\prime}(0) a_{1}^{2}(2 \pi / \cos \theta)=a_{1}(2 \pi / \cos \theta) a_{1}^{\prime}(2 \pi / \cos \theta)$. But $\left|a_{1}(2 \pi / \cos \theta)\right|=|J(2 \pi / \cos \theta)|$ cannot cancel, and we get $a_{1}^{\prime}(0) a_{1}(2 \pi / \cos \theta)=a_{1}^{\prime}(2 \pi / \cos \theta)$. Using the periodicity 
of $a_{1}^{\prime}$ and (5) we obtain

$$
a_{1}^{\prime}(0)\left(1-a_{1}(2 \pi / \cos \theta)\right)=\frac{2 \pi \tan ^{2} \theta}{\cos \theta}\left(a_{1}^{\prime}(0)\right)^{2}=0 .
$$

Thus, $a_{1}^{\prime}(0)=0$ and, using (5) again, $a_{1}$ is periodic. This proves the proposition.

It is important to note that the proof of the proposition above actually shows that $\kappa\left(Z^{h}\right)=0$, which will be essential in the proof of the next proposition.

Proposition 4. If $\mathcal{F}$ is a one-dimensional metric foliation on $H^{2 n+1}$, then $\mathcal{Z}$ makes a constant angle with $\mathcal{V}$ along $L$, for any leaf $L$.

Proof. Let $Z=Z^{v}+Z^{h} \in \mathcal{V} \oplus \mathcal{H}$ and let us assume that $\mathcal{V}$ makes an angle different from 0 or $\pi / 2$ with $\mathcal{Z}$ at some point on $L$. Let $V=\frac{1}{\left|Z^{v}\right|} Z^{v}$. We have the following:

$$
\begin{aligned}
V\langle V, Z\rangle & =\left\langle\nabla_{V} V, Z\right\rangle+\left\langle V, \nabla_{V} Z\right\rangle \\
& =\left\langle\nabla_{V} V, Z^{h}\right\rangle+\left\langle\nabla_{V} V, Z^{v}\right\rangle=\kappa\left(Z^{h}\right)=0,
\end{aligned}
$$

where the second equality follows from the fact that $Z$ is a Killing vector field and the third one follows from $\left\langle\nabla_{V} V, Z^{v}\right\rangle=\left|Z^{v}\right|\left\langle\nabla_{V} V, V\right\rangle=0$. The last equality follows from Proposition 3. Hence $\langle V, Z\rangle$ is constant along $L$.

The other two cases follow as a consequence of the case above.

Proposition 5. Let $\mathcal{F}$ be a one-dimensional metric foliation on $H^{2 n+1}$ and let $X$ be a basic vector field along a leaf $L$. Then, along $L, X$ makes a constant angle with the central direction $\mathcal{Z}$.

Proof. We may assume that $\mathcal{Z}$ is not tangent to $L$ since, by Proposition 4 , the conclusion is true if $\mathcal{Z}$ is tangent to $L$. Note that it is enough to prove the proposition for basic vector fields that are orthogonal to $\mathcal{Z}$ at some point $p \in L$. Indeed, let $X_{1}, \ldots, X_{2 n-1}, H$ be (local) basic fields along $L$ such that $\left\{X_{1}(p), \ldots, X_{2 n-1}(p)\right.$, $H(p)\}$ is an orthonormal basis of $\mathcal{H}_{p}$, where $X_{i}(p) \perp Z(p), H(p)=\frac{1}{\left|Z^{h}(p)\right|} Z^{h}(p)$, and $Z \in \mathcal{Z},|Z|=1$. As we will show below, $X_{i}$ remains orthogonal to $Z$ along $L$ for any $1 \leq i \leq 2 n-1$, and it follows that $H(q)=\frac{1}{\left|Z^{h}(q)\right|} Z^{h}(q)$ for any $q \in L$. Using the fact that $\mathcal{Z}$ makes a constant angle with $\mathcal{V}$ (and, thus, with $\mathcal{H}$ ), we may conclude that $\left|Z^{h}\right|$ is constant along $L$. Hence, since $H$ is basic, we may conclude that $Z^{h}$ is basic as well. The result follows easily since any unit basic vector field along $L$ can be written as $X=a Z^{h}+\sum_{i=1}^{2 n-1} a_{i} X_{i}$, where $a$ and $a_{i}$ are constants. Consequently, $\mid \cos \left(\left.\measuredangle(X, \mathcal{Z})|=| a|| Z^{h}\right|^{2}\right.$ is constant.

Now let $X$ be a basic vector field along $L$ such that $X_{p} \perp Z$ for some $p \in L$. Let us show that $X$ is orthogonal to $\mathcal{Z}$ along $L$. If $\tilde{p}$ is another point of $L$, consider the horizontal geodesics $\gamma$ and $\tilde{\gamma}$ starting at $p$ and $\tilde{p}$ in direction $X_{p}$ and $X_{\tilde{p}}$, respectively. By contradiction, assume that $X_{\tilde{p}}$ is not orthogonal to $\mathcal{Z}$ and let $J$ and $\tilde{J}$ be holonomy Jacobi vector fields along $\gamma$ and $\tilde{\gamma}$. Using the form of the Jacobi vector fields along geodesics orthogonal to $\mathcal{Z}$ given in 3.2(i), it is easy to see that, as $t \rightarrow \infty, \cos (\measuredangle(J(t), \mathcal{Z}))$ converges to some $\alpha \in[0,1]$. But, by Proposition $3, t \rightarrow \cos (\measuredangle(\tilde{J}(t), \mathcal{Z}))$ is a periodic function. This is a contradiction since, by Proposition $4, J(t)$ and $\tilde{J}(t)$ make the the same angle with $\mathcal{Z}$.

Let $\gamma$ be a unit speed horizontal geodesic with $\gamma(0)=e, \dot{\gamma}(0)=X \perp \mathcal{Z}$ and let $J$ be a Jacobi vector field along $\gamma$. As noted above, the coefficient of $Z$ in the 
expression of $J$ is a polynomial of degree at most three. In fact,

Lemma 2. If $J$ is a holonomy Jacobi vector field along a horizontal geodesic $\gamma$ with $\dot{\gamma}(0)=X \perp \mathcal{Z}$, then the coefficient of $Z$ in the expression of $J$ is a polynomial of degree at most two.

Proof. Let $0 \leq \theta_{0} \leq \pi / 2$ be the angle made by $\mathcal{V}_{e}$ with $\mathcal{Z}^{\perp}$, and note that we may assume $0<\theta_{0}<\pi / 2$. Indeed, if $\theta_{0}=0$ or $\pi / 2$, unless the coefficient of $Z$ in the expression of $J$ is constant (in which case we are done), there exists $t$ such that the vertical space at $\gamma(t)$ makes an angle different from 0 or $\pi / 2$ with $\mathcal{Z}$. If we left translate by $\gamma(-t)$, the problem is reduced to the case above.

Let $V=-\cos \theta_{0} U+\sin \theta_{0} Z$ be a unit vertical vector at $e$, where $U \perp Z$ and $|U|=1$. For each $\theta_{0} \leq \theta<\pi / 2$, consider the geodesic $\gamma_{\theta}$ with $\gamma_{\theta}(0)=e$ and $\gamma_{\theta}^{\prime}(0)=$ $\cos \theta Z+\sin \theta X_{1, \theta}$, where $X_{1, \theta}=\cos \phi X+\sin \phi U$ and $\phi=\sin ^{-1}\left(\tan \theta_{0} / \tan \theta\right)$. It is easy to see that $\gamma_{\theta}$ is a horizontal geodesic making an angle $\theta$ with $\mathcal{Z}$. Moreover, as $\theta \rightarrow \pi / 2, \gamma_{\theta}^{\prime}(0) \rightarrow X$.

Consider the holonomy Jacobi vector fields $J_{\theta}$ along $\gamma_{\theta}$ with $J_{\theta}(0)=V$. Based on the form of the Jacobi vector fields and Proposition 3,

$$
J_{\theta}=\sum_{k=1}^{n}\left(a_{k, \theta} E_{k, \theta}+\bar{a}_{k, \theta} \bar{E}_{k, \theta}\right)
$$

where

$$
\begin{gathered}
a_{1, \theta}(t)=a_{1, \theta}(0)+\bar{a}_{1, \theta}^{\prime}(0) \frac{1-\cos (\cos \theta t)}{\cos ^{2} \theta}+a_{1, \theta}^{\prime}(0) \frac{\sin (\cos \theta t)}{\cos \theta}, \\
\bar{a}_{1, \theta}(t)=\bar{a}_{1, \theta}(0)+\bar{a}_{1, \theta}^{\prime}(0) \frac{\sin (\cos \theta t)}{\cos \theta}-a_{1, \theta}^{\prime}(0) \frac{1-\cos (\cos \theta t)}{\cos \theta}, \\
E_{1, \theta}(t)=\cos \theta e^{t j(Z \cos \theta)} X_{1, \theta} \circ \gamma(t)-\sin \theta Z \circ \gamma(t), \\
\bar{E}_{1, \theta}(t)=e^{t j(Z \cos \theta)} Y_{1, \theta} \circ \gamma(t) .
\end{gathered}
$$

For $k \geq 2, a_{k, \theta}, \bar{a}_{k, \theta}, E_{k, \theta}$ and $\bar{E}_{k, \theta}$ are given by formulas similar to (4) and (6).

It is easy to check that since $J_{\theta} \rightarrow J$ as $\theta \rightarrow \pi / 2$, we get

$$
\langle J(t), Z\rangle=\lim _{\theta \rightarrow \pi / 2}\left\langle J_{\theta}(t), Z\right\rangle=\lim _{\theta \rightarrow \pi / 2} a_{1, \theta}(t) \sin \theta=\alpha_{1}+\frac{1}{2} \bar{\beta}_{1} t^{2}+\beta_{1} t,
$$

where $\alpha_{1}, \beta_{1}$ and $\bar{\beta}_{1}$ are the limits of $a_{1, \theta}(0), a_{1, \theta}^{\prime}(0)$ and $\bar{a}_{1, \theta}(0)$, respectively.

Theorem 1. Let $\mathcal{F}$ be a one-dimensional foliation on $H^{2 n+1}$ equipped with a left invariant metric. $\mathcal{F}$ is metric if and only if it is homogeneous.

Proof. Let $p$ and $\tilde{p}$ be two points on the same leaf. Also, let $X$ and $\tilde{X}$ be the values of a basic vector field at $p$ and $\tilde{p}$, respectively. We want to show that $\kappa(X)=\kappa(\tilde{X})$. Observe that $X$ (and $\tilde{X}$ ) may be chosen to be orthogonal to $\mathcal{Z}$ since, for $Z \in \mathcal{Z}$ with $|Z|=1$, we already have that $Z^{h}$ is basic and $\kappa\left(Z^{h}\right)$ is constant along individual leaves.

Let $\gamma$ and $\tilde{\gamma}$ be horizontal geodesics with $\dot{\gamma}(0)=X$ and $\dot{\tilde{\gamma}}(0)=\tilde{X}$. Recall that if $J$ is the holonomy Jacobi field along $\gamma$ with $|J(0)|=1$, then, by (8), we have $\kappa(X)=-\left\langle J^{\prime}(0), J(0)\right\rangle$. Using Proposition 4 and 3.2(i), we obtain

$$
\frac{|f(t)|}{|J(t)|}=|\cos (\measuredangle(J(t), Z))|=|\cos (\measuredangle(\tilde{J}(t), Z))|=\frac{|\tilde{f}(t)|}{|\tilde{J}(t)|},
$$


where $\tilde{J}$ is the holonomy Jacobi field along $\tilde{\gamma}$ with $|\tilde{J}(0)|=1$. The relation above implies

$$
f^{2}(t)|\tilde{J}(t)|^{2}=\tilde{f}^{2}(t)|J(t)|^{2}
$$

Let us denote $|J(t)|^{2}$ by $h(t)$ and $|\tilde{J}(t)|^{2}$ by $\tilde{h}(t)$. Note that $h$ and $\tilde{h}$ cannot have any real roots since a holonomy Jacobi field cannot have any zeroes. Also note that $f$ and $\tilde{f}$ are polynomials of degree at most two, while $h$ and $\tilde{h}$ have degree at most four.

We will show that $h=\tilde{h}$, provided that $f$ is not identically zero. Note that $h=\tilde{h}$ is enough to conclude that $\kappa$ is basic since $\kappa(X)=-h^{\prime}(0)$ and $\kappa(\tilde{X})=-\tilde{h}^{\prime}(0)$. If $f$ is identically zero, in order to show that $\kappa$ is basic we will adapt the technique used in Theorem 1.1 from [3]. First, assume $f$ is not identically zero and consider the following cases:

(1) $f$ and $\tilde{f}$ are not relatively prime.

By (8) and the observations above, we must have $f=a \tilde{f}$, for some real number $a$. Indeed, since $f$ and $\tilde{f}$ are not relatively prime, they must have the same degree, since otherwise either $h$ or $\tilde{h}$ will have a zero. For the same reason, the common factor of $f$ and $\tilde{f}$ must be of degree two. Thus $f=a \tilde{f}$, which implies $\tilde{h}=h / a^{2}$. Since $h(0)=\tilde{h}(0)=1$, we have $h=\tilde{h}$.

(2) $f$ and $\tilde{f}$ are relatively prime.

In this case we get that $f^{2}$ divides $h$. Consequently, $f^{2}$ divides $h-f^{2}$. Note that if $h(0)=f^{2}(0)$, then the $\mathcal{Z}$ is vertical at $p$ and the leaf through $p$ is the geodesic in the central direction along which $\kappa$ is basic. If $h(0) \neq f^{2}(0)$, then the degree of $h-f^{2}$ is less than or equal to two. By degree count, $f$ must have degree at most one. But $f$ cannot have degree one, since $f^{2}$ divides $h$ and $h$ has no real roots. Thus, $f$ is constant. A similar argument shows that $\tilde{f}$ must be constant. If $f \neq 0$, as before, we obtain $h=\tilde{h}$.

Let us discuss the case when $f$ is identically zero. By 3.2(i) and (8), $g, \tilde{f}$, and $\tilde{g}$ are also identically zero. This implies that the vertical bundles at $p$ and $\tilde{p}$ are orthogonal to $\operatorname{span}\{X, j X, Z\}$ and $\operatorname{span}\{\tilde{X}, j \tilde{X}, Z\}$, respectively.

Let $\lambda=\kappa(X)$, and consider the vector field $J_{0}(t)=(1-\lambda t) E \circ \gamma(t)$ along $\gamma$, where $E$ is the left invariant vector field defined by the condition that $E_{p}$ is vertical of unit length. Based on the observations above, $J_{0}$ is a Jacobi vector field. Moreover, $J_{0}^{\prime}(0)=-\lambda J_{0}(0)=-S_{\dot{\gamma}(0)} J_{0}(0)$. Thus, if $\pi$ is the Riemannian submersion locally defining $\mathcal{F}, J_{0}$ projects to a Jacobi field $\pi_{*} J_{0}$ along $\pi \circ \gamma$ (see [7]). Assuming $\lambda \neq 0, \pi \circ \gamma$ has a conjugate point at $1 / \lambda$. By Lemma 1 in [7, there exists a unique Jacobi field $\tilde{J}_{0}$ along $\tilde{\gamma}$ such that $\pi_{*} J_{0}=\pi_{*} \tilde{J}_{0}, \tilde{J}_{0}(1 / \lambda)=0$, and $\tilde{J}_{0}^{\prime v}+S_{\dot{\tilde{\gamma}}} \tilde{J}_{0}^{v}+A_{\dot{\tilde{\gamma}}} \tilde{J}_{0}^{h}=0$. But $\tilde{J}_{0}=(1-\lambda t) \tilde{E} \circ \tilde{\gamma}$, where $\tilde{E}$ is the left invariant vector field for which $\tilde{E}_{\tilde{p}}$ is vertical and of length one. To see this, recall that $\tilde{J}_{0}(0)$ is orthogonal to $\tilde{X}, j \tilde{X}$, and $Z$. Thus, the coefficients of $Z$ and $\tilde{Y}=j \tilde{X}$ in the expression of $\tilde{J}_{0}$ from $3.2(\mathrm{i})$ will cancel at $t=0$. Since they also cancel at $t=1 / \lambda$, they must be identically zero. Based on 3.2(i) again and the fact that $\tilde{J}_{0}$ cancels at $t=1 / \lambda$, we may conclude that $\tilde{J}_{0}$ has the form above. Now, if we let $t=0$ in $\tilde{J}_{0}^{\prime v}+S_{\dot{\tilde{\gamma}}} \tilde{J}_{0}^{v}+A_{\dot{\tilde{\gamma}}} \tilde{J}_{0}^{h}=0$, we get $\kappa(\tilde{X})=-\left\langle S_{\tilde{X}} \tilde{J}_{0}(0), \tilde{J}_{0}(0)\right\rangle=\lambda\left|\tilde{J}_{0}(0)\right|^{2}$. Consequently, $\kappa(\tilde{X})=\lambda$. In view of the above, the same must be true if $\lambda=0$. Consequently $\kappa$ is basic, and we are done. 
In 10, G. Walschap shows that every codimension one metric foliation on $H^{2 n+1}$ is actually left invariant and is generated by an ideal of the Lie algebra $\mathfrak{h}_{2 n+1}$. Thus, the foliation is given by cosets of the form $\left\{g K, g \in H^{2 n+1}\right\}$, where $K$ is a normal subgroup of $H^{2 n+1}$. As such, all these foliations are homogeneous.

Using the result mentioned above in conjunction with Theorem 1, we obtain the following:

Theorem 2. All metric foliations on $H^{3}$ are homogeneous.

The next theorem generalizes a result of G. Walschap ([9]) regarding one-dimensional metric foliations on three-dimensional nilmanifolds.

Theorem 3. Let $\Gamma$ be a lattice in $H^{2 n+1}$. There exists a unique one-dimensional metric foliation $\mathcal{F}$ on $\Gamma \backslash H^{2 n+1} . \mathcal{F}$ is homogeneous and its lift to $H^{2 n+1}$ has vertical bundle $\mathcal{Z}$.

Proof. Let $\tilde{\mathcal{F}}$ be the lifted foliation. Note that $\tilde{\mathcal{F}}$ is a one-dimensional metric foliation on $H^{2 n+1}$. By Theorem 1 , any such foliation is homogeneous. But then $\mathcal{F}$ is also homogeneous, since the isometric action defining $\tilde{\mathcal{F}}$ descends to an isometric action on $\Gamma \backslash H^{2 n+1}$.

According to [1], the identity component of $\operatorname{Iso}\left(\Gamma \backslash H^{2 n+1}\right)$ is $C / C \cap \Gamma$, where $C$ is the center of $H^{2 n+1}$. If $\pi_{1}: C \rightarrow C / C \cap \Gamma$ and $\pi_{2}: H^{2 n+1} \rightarrow \Gamma \backslash H^{2 n+1}$ denote the projection maps, then the action of $C / C \cap \Gamma$ on $\Gamma \backslash H^{2 n+1}$ is given by $\left(\pi_{1}(c), \pi_{2}(h)\right) \rightarrow \pi_{2}(c h)$. Consequently, the leaves of the lifted foliation are orbits of $C$ acting on the left on $H^{2 n+1}$, and the conclusion follows. Note that we obtain a principal circle bundle over $T^{2 n}$.

Theorem 2 remains valid for metric foliations on $\Gamma \backslash H^{3}$. This is due to the fact that there are no two-dimensional metric foliations on $\Gamma \backslash H^{3}$. Indeed, if $\mathcal{F}$ is such a foliation, by Theorem 2, its lift must be homogeneous. But then $\mathcal{F}$ must also be homogeneous, and this is impossible because the dimension of the isometry group of $\Gamma \backslash H^{2 n+1}$ is one. The same type of argument used in conjunction with the homogeneity of metric foliations of codimension one on $H^{2 n+1}$ implies the following:

Proposition 6. Let $\Gamma$ be a lattice in $H^{2 n+1}$. There are no metric foliations of codimension one on $\Gamma \backslash H^{2 n+1}$.

\section{REFERENCES}

[1] P. Eberlein, Geometry of 2-Step Nilpotent Lie Groups with a Left Invariant Metric, Ann. scient. Éc. Norm. Sup. $4^{e}$ série, t. 27 (1994), 611-660. MR1296558 (95m:53059)

[2] D. Gromoll and K. Grove, One-Dimensional Metric Foliations on Constant Curvature Spaces, Differential Geometry and Complex Analysis, Springer-Verlag, Berlin, Heidelberg (1985), 165-168. MR0780042 (86b:53026)

[3] D. Gromoll and G. Walschap, Metric Fibrations on Euclidean Spaces, Asian J. Math 1 (1997), 716-728. MR.1621572 (99b:53041)

[4] D. Gromoll and G. Walschap, The Metric Fibrations of Euclidean Spaces, J. Differential Geometry 57 (2001), 233-238. MR.1879226 (2002k:53053)

[5] C. Jang and K. Park, Conjugate Points on 2-Step Nilpotent Groups, Geom. Dedicata 78 (2000), 65-80. MR1742200 (2001c:53067)

[6] B. O'Neill, The Fundamental Equations of a Submersion, Michigan Math. J. 13 (1966), 459-469. MR 0200865 (34:751)

[7] B. O'Neill, Submersions and Geodesics, Duke. Math. J. 34 (1967), 363-373. MR0216432 $(35: 7265)$ 
[8] P. Tondeur, Foliations on Riemannian manifolds, Springer-Verlag, New York (1988). MR0934020 (89e:53052)

[9] G. Walschap, Cut and Conjugate Loci in Two-Step Nilpotent Lie Groups, J. Geom. Anal. 7 (1997), 343-355. MR:1646788 (99m:53094)

[10] G. Walschap, Geometric Vector Fields on Lie Groups, Diff. Geom. Appl. 7 (1997), 219-230. MR1480535 (98g:53048)

[11] G. Walschap, Umbilic foliations and curvature, Illinois J. Math. 41 (1997), 122-128. MR 1433190 (97m:53053)

Department of Mathematics, Computer Science and Statistics, SUNy College at Oneonta, Oneonta, New York 13820

E-mail address: munteam@oneonta.edu 